\title{
Beam effect on electromagnetic ion-cyclotron waves with general loss - cone distribution function in an anisotropic plasma-particle aspect analysis
}

\author{
G. Ahirwar, P. Varma, and M. S. Tiwari \\ Department of Physics \& Electronics, Dr. H. S. Gour University, Sagar, (M.P.) 470003, India \\ Received: 1 July 2006 - Revised: 15 September 2006 - Accepted: 20 September 2006 - Published: 8 March 2007
}

\begin{abstract}
The effect of upgoing ion beam and temperature anisotropy on the dispersion relation, growth rate, parallel and perpendicular resonant energies, and marginal instability of the electromagnetic ion cyclotron (EMIC) waves, with general loss-cone distribution function, in a low $\beta$ homogeneous plasma, is discussed by investigating the trajectories of the charged particles. The whole plasma is considered to consist of resonant and non-resonant particles. The resonant particles participate in an energy exchange with the waves, whereas the non-resonant particles support the oscillatory motion of the waves. The effects of the steepness of the loss-cone distribution, ion beam velocity, with thermal anisotropy on resonant energy transferred, and the growth rate of the EMIC waves are discussed. It is found that the effect of the upgoing ion beam is to reduce the energy of transversely heated ions, whereas the thermal anisotropy acts as a source of free energy for the EMIC waves and enhances the growth rate. It is found that the EMIC wave emissions occur by extracting energy of perpendicularly heated ions in the presence of an upflowing ion beam and a steep loss-cone distribution function in the anisotropic magnetoplasma. The effect of the steepness of the loss-cone is also to enhance the growth rate of the EMIC waves. The results are interpreted for EMIC emissions in the auroral acceleration region.
\end{abstract}

Keywords. Ionosphere (Wave-particle interactions) - Magnetospheric physics (Auroral phenomena) - Space plasma physics (Waves and instabilities)

\section{Introduction}

Electromagnetic ion-cyclotron (EMIC) waves and the ion beam interactions take place in several space environments,

Correspondence to: M. S. Tiwari

(tiwarims@rediffmail.com) as well as in laboratory plasma. It is found that an inverted $-\mathrm{V}$ electron beam with an energy of $\mathrm{keV}$ can excite electromagnetic ion-cyclotron (EMIC) waves propagating parallel to the magnetic field with a frequency below the hydrogen gyrofrequency. The oxygen and hydrogen EMIC waves excited by the energetic electron beam might explain the low frequency electric and magnetic field noise $(\sim 100 \mathrm{~Hz})$ in the auroral zone. Ionospheric electrons trapped or reflected by EMIC waves are accelerated to energies of several $\mathrm{keV}$, as described by Temerin et al. (1986).

The importance of ion cyclotron waves in auroral physics lies in their ability to heat and accelerate ions, and perhaps to provide anomalous resistivity, allowing the creation of a parallel potential drop. Since such processes have global implications for the magnetosphere, these waves have been the subject of numerous publications (Kennel and Petschek, 1966; Temerin and Lysak, 1984; Cattell et al., 1991; McFadden et al., 1998a; Erlandson and Zanetti, 1998) and show the existence of electromagnetic waves. Chaston et al. (1998) have shown that these waves can have a Poynting flux directed upward out of the auroral oval, suggesting that the waves observed at higher altitudes may have their source at the base of the auroral potential structure where they are electromagnetic.

Observations suggesting $\mathrm{H}_{e}^{+}$energization by electromagnetic ion-cyclotron (EMIC) waves in the magnetosphere have been reported (Mauk et al., 1981; Anderson and Fuselier, 1994; Fuselier and Anderson, 1996). The EMIC waves can be generated in the equatorial magnetosphere from the ion cyclotron instability driven by anisotropic $\left(\mathrm{T}_{\perp p} / \mathrm{T}_{\| p}\right)$ energetic $(10 \mathrm{keV}$ to $50 \mathrm{keV})$ proton populations. In the presence of low energy $\mathrm{H}_{e}^{+}$, these waves will interact resonantly with $\mathrm{H}_{e}^{+}$, heating it perpendicular to the magnetic field direction (Gendrin and Roux, 1980). In past the studies on EMIC instability, including $\mathrm{H}_{e}^{+}, \mathrm{O}^{+}$and their anisotropies, have been performed by various authors (Gomberoff and Neira,

Published by Copernicus GmbH on behalf of the European Geosciences Union. 
1983; Gomberoff and Elgueta, 1991; Gomberoff et al., 1996; Thorne and Horne, 1997; Horne and Thorne, 1997). In the present analysis, we have considered only $\mathrm{H}_{e}^{+}$to examine the behavior of EMIC waves for the auroral acceleration process.

Chaston et al. (2002) have stated that waves with frequencies in the vicinity of the proton cyclotron frequency and its harmonics are commonly observed from the Fast Auroral Snapshot spacecraft when traversing regions of auroral particle acceleration. In areas of upward current, large amplitude electromagnetic waves with frequencies within $5 \%$ of the local proton gyrofrequency $\Omega_{p}$ and its harmonics are often observed where an upstreaming ion beam exist (Chaston et al., 2002). The upgoing ion beams are reported by the S3-3 satellite (Shelley et al., 1976; Mozer et al., 1977) and by the Polar satellite data (Mozer and Hull, 2001). Ions are often observed to have been accelerated transversely to the background magnetic field in the auroral region (Sharp et al., 1977; Lund et al., 2001). As they drift upward in the diverging field, ions exchange some of their perpendicular energy via the mirror force for energy of motion along the field. Such distributions, known as ion conics, have their characteristic shape in velocity space (Lund et al., 2001). The association of ion conics with EMIC waves has been given by various workers (Thorne and Horne, 1997; Horne and Thorne, 1997; Lund et al., 1999a, b, c). Here we concentrate upon the excitation of EMIC waves by an upflowing ion beam in the converging magnetic field of the auroral acceleration region and the energy exchange of the ions along and perpendicular to the magnetic field.

The effect of loss-cone distribution is to enhance the growth rate of EMIC waves, as well as to increase the energies of the resonant ions in the presence of the waves (Ahirwar et al., 2006). The transversely accelerated ions and their association with EMIC waves in the auroral acceleration region have been recently reported by various workers (Lund et al., 1999a, 2000, 2001; Chaston et al., 2002) in the analysis of the FAST satellite data. The equilibrium dipolar magnetic field of the Earth is curved in a meridonial plane and may introduce loss-cone effects in the particle distribution function (Varma and Tiwari, 1992; Tiwari and Varma, 1991, 1993; Dwivedi et al., 2001b; Ahirwar et al., 2006).

In most of the theoretical work reported so far, the velocity distribution function has been assumed to be either ideally Maxwellian or bi-Maxwellian, ignoring the steep loss-cone feature. Plasma in mirror-like devices and in the auroral region with curved and converging field lines, depart considerably from a Maxwellian distribution and have a steep losscone distribution (Dwivedi et al, 2001a; Duan et al., 2005). In this paper we will discuss the effect of the general losscone distribution function on the EMIC waves in the presence of ion beams and thermal anisotropy. The main objective of the present investigation is to examine the effect of ion beams and thermal anisotropy on EMIC waves at different loss-cone indices $J$, in view of the observations in the auroral acceleration region. The present analysis is based on
Dawson's (1961) theory of Landau damping, which was further extended by Terashima (1967), Dwivedi et al. (2002), Mishra and Tiwari (2006) and Ahirwar et al. (2006).

The method adopted, known as particle analysis, has been widely used in the analysis of electrostatic and electromagnetic instabilities (Tiwari and Varma,1991; Dwivedi et al., 2001a, b, 2002; Duan et al., 2005; Mishra and Tiwari, 2006; Ahirwar et al., 2006). The relative importance of this approach over fluid and kinetic approachs is also discussed (Varma and Tiwari, 1992, 1993; Tiwari and Varma, 1993). The main advantages of this approach is to consider the energy transfer between waves and particles, along with the discussion of waves dispersion and growth/damping rate of the waves. The method may be suitable to deal with the auroral electrodynamics where particle acceleration is also important alongwith waves emissions. The results obtained by this approach are the same as those derived using a kinetic approach.

The main feature of our study is to show the effect of an upgoing ion beam and thermal anisotropy on EMIC waves with a general loss-cone distribution function in a low $\beta$ homogeneous plasma and discuss this effect by investigating the trajectories of the charged particles. The term "losscone" is added, as it has been widely adopted in literature; therefore, it is called the general loss-cone distribution function, otherwise it is not truly general. More specifically, it is known as the Dory-Guest-Harris distribution and is the most frequently used loss-cone distribution in the plasma physics literature. Its loss-cone region, $\frac{\partial f}{\partial V_{\perp}}>0$, is given by $\frac{V_{\perp}}{V_{T \perp}}<J^{1 / 2}$ (Summers and Thorne, 1995). The applicability and importance of this distribution have been discussed in detail by Summers and Thorne (1995, and references therein). According to them a loss-cone distribution is a distribution that has a deficiency of particles for small values of $V_{\perp}$, or small values of pitch angle $\alpha$, defined by $\alpha=\tan ^{-1}\left(\frac{V_{\perp}}{V_{\amalg}}\right)$; it is usually the case that for a loss-cone distribution there is a region of velocity $\left(\mathrm{V}_{\perp}, \mathrm{V}_{\Pi}\right)$ space, such that $\frac{\partial f}{\partial V_{\perp}}>0$. In practice, a geometrical loss-cone region can not be completely empty $\left(\mathrm{V}_{\perp}=0\right)$, on account of particle scattering and collisional processes (Summers and Thorne, 1995). Loss-cone distributions typically arise when a plasma is confined in a magnetic trap (or bottle), such as those occurring in the auroral ionosphere (Gomberoff and Cuperman, 1981). To our knowledge, however, the general loss-cone distribution has not yet been observed in the auroral acceleration region, but the converging magnetic field exhibiting mirror-like geometry, and the interplay between the magnetic mirror force and the parallel electric field are the motivations to adopt the losscone distribution in our study. The various plasma instability processes also may lead to the loss-cone distribution function (Horne and Thorne, 2000). Horne and Thorne (1997) have found a correlation between periods of EMIC waves activity and pitch angle distributions, which resembles an " $\mathrm{X}$ " in velocity space and may be the signature of loss-cone 
distributions. The ion beam measured by Mozer and Hull (2001) has been considered to enter the plasma which follows the loss-cone distribution function. The results are compared with those derived by Kennel and Petschek (1966). They have stated that for the particle energies greater than $\frac{B^{2}}{8 \pi N}$, the interaction of ion cyclotron noise with ions leads to diffusion in the pitch angle. They observed that the steady-state pitch angle distribution, subject to the boundary condition that particles are lost from the loss cone, has an almost constant anisotropy which has the appropriate sign to be unstable. The resultant growth rate of the waves is then directly proportional to the number of resonant particles.

The EMIC waves generated in the equatorial magnetosphere travel towards the auroral ionosphere in the converging magnetic field; thus, it is assumed that the distribution may depart from ideally Maxwellian and allow for a general loss-cone distribution function (Duan et al., 2005; Mishra and Tiwari, 2006; Ahirwar et al., 2006). In the past the mirror structure for the development of the quasi-static potential drop along auroral field lines has been adopted by various authors (e.g. Schriver et al., 2003). The various plasma instability processes may also lead to the loss-cone distribution (Horne and Thorne, 2002). The mirror geometery is the justification of their choice and the experimental evidence is not known, to our knowledge. The ion beam considered does not follow the loss-cone distribution; it is the background plasma of the auroral acceleration which may permit the general loss-cone distribution, due to converging magnetic field lines. The electron beam may generate the EMIC waves, as mentioned in the Introduction. The ion beam in the direction of the wave motion may damp these waves, however, the ion beam opposite to the wave motion may excite the waves, as reported in this paper. In our present paper, we have considered the electrodynamics of the auroral ionospheric region by an EMIC wave study. Lund et al. (2000) predicted some observational evidences through satellite data. Ion conics may enhance the growth rate of EMIC waves at the ionospheric region, which provides the potential of our theoretical model to study EMIC wave characteristics in the auroral acceleration region (Ahirwar et al., 2006). The EMIC turbulence plays an important role in the loss-cone current potential relationship. It has been suggested that the loss-cone effects can enhance the anomalous resistivity for a given turbulence level. Since the steep loss-cone distribution in the presence of EMIC waves and the ion beam enhances the growth rate, the anomalous resistivity and transport resulting from this instability are likely to play a crucial role in the auroral acceleration region. The conversing magnetic field lines in the higher latitude auroral ionosphere may be considered suitable for the use of the generalized loss-cone distribution function. An upflowing ion beam, along with energetic particles may excite EMIC waves. Both the ion and electrons are assumed to follow the general loss-cone distribution function.

\section{Mathematical considerations}

The waves propagating in the direction of the ambient magnetic field along the $\mathrm{z}$-axis are considered. The whole plasma has been considered to consist of resonant and non-resonant particles. The resonant particles participate in an energy exchange with the waves, whereas the non-resonant particles support the oscillatory motion of the waves. The EMIC waves are assumed to start at $\mathrm{t}=0$ when the resonant particles are not yet disturbed. The trajectories of the particles are then evaluated within the framework of linear theory. Taking the particle trajectory in the presence of EMIC waves, the dispersion relation, the change in the charged particle energy and the growth rate are then derived for different distribution indices in the presence of upflowing ion beams, in an anisotropic plasma by the particle aspect analysis.

The basic mathematical treatment of EMIC waves using the particle aspect approach is given by Misra and Tiwari (1979). The left-handed circularly polarized EMIC waves, having an angular frequency $\omega$, are considered as:

$B_{x}=B \cos (k z-\omega t)$
$B_{y}=B \sin (k z-\omega t)$.

When the system is co-moving with the waves, the electric field vanishes. Thus, the wave magnetic field has the form

$B=B_{x}[\cos k z] \boldsymbol{x}+B_{y}[\sin k z] \boldsymbol{y}$,

where the following transformation has been performed:

$z^{\text {wave }}=z^{\text {lab }}-(\omega / k) t$
$v^{\text {wave }}=v^{\text {lab }}-(\omega / k)$.

Since ck $/ \omega \gg 1$, the magnetic field amplitude may be regarded in both systems as identical. Using the equation of ion motion in the presence of the wave, Misra and Tiwari (1979) have derived the ion perturbed velocities as

$$
\begin{aligned}
\delta V_{\perp}= & \frac{h \Omega\left(V_{\prod_{0}}-\frac{\omega}{k}\right)}{k V_{\amalg 0}-(\omega-\Omega)}[\cos (k z-\omega t-\psi) \\
& \left.-\varepsilon \cos \left(k z-\omega t-\psi-\left(k V_{\prod_{0}}-(\omega-\Omega)\right) t\right)\right] \\
\delta V_{\Pi}= & \frac{-h V_{\perp 0} \Omega}{k V_{\amalg 0}-(\omega-\Omega)}[\cos (k z-\omega t-\psi \\
& \left.-\varepsilon \cos \left(k z-\omega t-\psi-\left(k V_{\prod_{0}}-(\omega-\Omega)\right) t\right)\right],
\end{aligned}
$$

where $\mathrm{V}_{\perp 0}, \mathrm{~V}_{I I 0}$ are the initial values of the velocities at $\mathrm{t}=\mathrm{o}$, $\mathrm{z}=\mathrm{z}_{0}+\mathrm{V}_{\|} \mathrm{t}$ and $\psi=\psi_{0}-\Omega \mathrm{t}$. $\varepsilon=0,1$ for the non-resonant and resonant particles and $h=\frac{B}{B_{0}}$ is the ratio of electromagnetic to static magnetic field amplitude. $\Omega=\frac{q B_{0}}{m c}$ is the ion cyclotron frequency and $\psi$ is the initial phase in perpendicular velocity and $\psi_{0}=\psi(\mathrm{t}=0)$. 


\subsection{Distribution function}

To determine the dispersion relation and the growth rate, the density distribution is defined as

$N(y, \bar{V})=N_{0} f_{\perp}\left(V_{\perp}\right) f_{\prod}\left(V_{\Pi}\right)$,

where we consider a general loss-cone distribution function for $f_{\perp}\left(V_{\perp}\right)$ as (Gomberoff and Cuperman, 1981; Summers and Thorne, 1995; Tiwari and Varma, 1991, 1993; Varma and Tiwari, 1992; Ahirwar et al., 2006)

$f_{\perp}\left(V_{\perp}\right)=\left[\frac{V_{\perp}^{2 J}}{\pi V_{T \perp}^{2(J+1)} J !}\right] \exp \left(-\frac{V_{\perp}^{2}}{V_{T \perp}^{2}}\right)$

and $f_{\Pi}\left(V_{\Pi}\right)$, which is defined by the drifting Maxwellian (Varma and Tiwari, 1992)

$f_{\Pi}\left(V_{\Pi}\right)=\left(\frac{1}{\sqrt{\pi} V_{T \Pi}}\right) \exp \left\{-m\left(V_{\Pi}-V_{D}\right)^{2} / 2 T_{\Pi}\right\}$,

where $j$ is the distribution index and measures the steepness of the loss-cone feature (Tiwari and Varma, 1991, 1993; Varma and Tiwari, 1992). In the case of $J=0$ this represents a bi-Maxwellian distribution and for $J=\infty$ this reduces to the Dirac delta function (Tiwari and Varma, 1993). $V_{T}^{2}=\frac{2 T}{m}$ and $V_{T \perp}^{2}=(J+1)^{-1} \frac{2 T_{\perp}}{m}$ are the squares of the parallel and perpendicular thermal velocities with respect to the external magnetic field. $V_{D}$ defines the beam velocity of the particles.

The density perturbation associated with the velocity perturbation is obtained as (Misra and Tiwari, 1979; Ahirwar et al., 2006)

$n_{1}=\frac{h V_{\perp 0} \Omega k N(V)}{\left[k V_{\prod 0}-(\omega-\Omega)\right]^{2}}\left[\cos \chi-\varepsilon \cos \chi_{0}+\varepsilon t \Lambda \sin (\chi-\Lambda t)\right]$,

where $\chi=\mathrm{kz}-\omega \mathrm{t}-\psi$ and $\Lambda=\left(\mathrm{kV}_{\| 0}-(\omega-\Omega)\right)$.

\subsection{Dispersion relation}

Since the existence of the ion energy anisotropy is an established fact and growth is only possible when $\frac{V_{T \perp}^{2}}{V_{T}^{2}}>1$, we are interested in the behavior of those particles for which $\frac{V_{T \perp}^{2}}{V_{T}^{2}}>1$. We consider the cold plasma dispersion relation for the EMIC waves as (Misra and Tiwari, 1979; Ahirwar et al., 2006)

$\frac{c^{2} k^{2}}{\omega^{2}}=\left(\frac{\omega_{p i}^{2}}{\Omega^{2}}\right)\left(1-\frac{\omega}{\Omega}\right)^{-1}$,

where $\omega_{p i, e}^{2}=\frac{4 \pi N_{0} e^{2}}{m_{i, e}}$ is the plasma frequency for the ions.

We have considered a background plasma consisting of electrons and non-drifting ions relative to the electrons. It is assumed that ions and electrons of the background plasma follow the loss-cone distribution function. A beam of ions of very low density, in comparison to the background plasma density streaming with constant velocity along the magnetic field is considered. Thus, a drifting Maxwellian distribution function along the magnetic field for the background plasma ion density is assumed. The possibilities of EMIC wave excitation and particle heating in such a plasma in the presence of an ion beam are explored. It is a current free plasma.

The dispersion relation for electromagnetic ion-cyclotron waves propagating in the direction of the external magnetic field, in a system consisting of electrons, proton core and proton beam is given in Gomberoff and Elgueta (1991); Gomberoff (2003). If the beam density relative to core density is small, the dispersion relation reduces to a cold plasma dispersion relation, as we have adopted. This approach may be analogous to the test-particle approach adopted by Dusenbery and Hollweg (1981). In this paper we have not considered the transfer of energy and momentum through EMIC waves back to the ion beam. The non-drifting ions, consisting of resonant and non-resonant particles, participate in the energy exchange with the wave. The cold plasma dispersion relation approximation is also a good approximation to the warm plasma dispersion relation, provided that plasma $\beta<1$ (Gomberoff and Elgueta, 1991). Thus, the non-drifting ions are treated as anisotropic and the wave growth is possible. For the large ion beam density, one must consider the dispersion effects of the beam, which does change the dispersion relation (Gomberoff and Elgueta, 1991; Gomberoff, 2003). Only when the drift velocity becomes very large, do the EMIC and the beam contribution to the dispersion relation decouple, but then the drifting ions are not influenced by the original cyclotron wave. In our treatment we have assumed that the ion beam is not affected by EMIC waves.

\subsection{Wave energy density and growth rate}

The wave energy density $\mathrm{W}_{w}$ per unit wavelength is the sum of the pure field energy $U$ and the changes in the energy of the non-resonant particles $W i$, i.e. the total energy per unit wavelength is given as (Misra and Tiwari, 1979)

$W_{w}=U+W i$,

where the energy of electromagnetic wave $U$ is defined by the expression (Misra and Tiwari, 1979; Ahirwar et al., 2006)

$U=\left(\frac{1}{16 \pi}\right)\left[\left(\frac{d}{d \omega}\right)\left(\omega \varepsilon_{i k}\right) E_{1}^{*} E_{k}+|B|^{2}\right]$,

where $\varepsilon_{i k}$ is the dielectric tensor. After the calculation, the electromagnetic wave energy per unit wavelength is given by

$U=\left(\frac{\lambda B^{2}}{8 \pi}\right)\left[\frac{(2 \Omega-\omega)}{(\Omega-\omega)}\right]$. 
The change in energy of non-resonant particles in the presence of an EMIC wave is given by

$$
\begin{aligned}
W_{i}= & \int_{0}^{\lambda} d z \int_{0}^{2 \pi} d \psi \int_{0}^{\infty} V_{\perp} d V_{\perp} P \\
& \int_{-\infty}^{\infty} d V_{\prod 0} \frac{m}{2}\left[\left(N+n_{1}\right)(V+\delta V)^{2}-N V^{2}\right] .
\end{aligned}
$$

\subsection{Non-resonant energy}

Using Eqs. (4), (5), (8) and (13) with $\varepsilon=0$ we find the parallel non-resonant energy as (Misra and Tiwari, 1979)

$$
\begin{aligned}
W_{i \prod}= & \frac{-\lambda B^{2}}{8 \pi} \frac{C_{J}}{V_{T \prod}^{2}} \frac{\omega_{p i}^{2}}{c^{2} k^{2}}\left[\frac{1}{2} Z_{1}\left(\zeta-\frac{V_{D}}{V_{T} \Pi}\right)\right. \\
& \left.+\frac{\omega-\Omega}{k_{\prod} V_{T \prod}} Z_{2}\left(\zeta-\frac{V_{D}}{V_{T \Pi}}\right)\right]
\end{aligned}
$$

and perpendicular non-resonant energy as

$$
\begin{aligned}
W_{i \perp}= & \frac{\lambda}{2} \frac{B^{2}}{8 \pi} \frac{\omega_{p i}^{2}}{c^{2} k^{2}}\left[D _ { J } \left(1-\frac{2 \Omega}{k_{\Pi} V_{T} \Pi} Z\left(\zeta-\frac{V_{D}}{V_{T \Pi}}\right)\right.\right. \\
& \left.+\frac{\Omega^{2}}{k^{2} V_{T}^{2}} Z_{1}\left(\zeta-\frac{V_{D}}{V_{T} \Pi}\right)\right)+\frac{2 C_{J}}{V_{T}^{2}} \\
& \left.\left(Z_{1}\left(\zeta-\frac{V_{D}}{V_{T}}\right)-\frac{\Omega}{k_{\Pi} V_{T}} Z_{2}\left(\zeta-\frac{V_{D}}{V_{T}}\right)\right)\right]
\end{aligned}
$$

The perpendicular (transverse) energy and the parallel resonant energy of the resonant ions are calculated with the help of Eqs. (4), (5), (8) and (13), with $\varepsilon=1$ as (Misra and Tiwari, 1979)

$$
\begin{aligned}
W_{r \perp}= & \frac{\pi^{3 / 2} B^{2} \omega_{p i}^{2}}{C^{2} k^{2}} \times \frac{\Omega_{i}^{2}}{\omega \cdot k^{2} V_{T} \Pi} \\
\Pi & {\left[(J+1) \frac{V_{T \perp}^{2}}{V_{T}^{2}}\left(\frac{\omega^{\prime}-\Omega_{i}}{\Omega_{i}}\right)^{2}\right] } \\
& \exp \left[-\frac{\left(\omega^{\prime}-\Omega_{i}\right)^{2}}{k^{2} V_{T}^{2} \Pi} \prod^{\Pi}\right. \\
W_{r \prod}= & \frac{\pi^{3 / 2} B^{2} \omega_{p i}^{2}}{C^{2} k^{2}} \times \frac{\Omega_{i}^{2}}{\omega \cdot k^{2} V_{T} \Pi} \\
\prod & {\left[\begin{array}{c}
\Pi \\
(J+1) \frac{V_{T \perp}^{2}}{V_{T}^{2}}\left(\frac{\omega^{\prime}-\Omega_{i}}{\Omega_{i}}\right)^{2}
\end{array}\right] }
\end{aligned}
$$

$$
\left.\exp \left[-\frac{\left(\omega^{\prime}-\Omega_{i}\right)^{2}}{k^{2} V_{T}^{2} \Pi}\right]_{\Pi}\right],
$$

where $\omega^{\prime}=\omega-k_{\prod} V_{D}$.

The above expressions represent the exchange of energy between waves and particles. Thus, the heating and acceleration of plasma particles are suitably described by the particle aspect approach. This approach also resolves the direction of the accelerations critically; therefore, it may be useful to explain the charged particle acceleration in the auroral ionosphere and plasma-sheet boundary layer with mirror-like geometries of the magnetic field lines.

Using the law of conservation of energy

$\frac{d}{d t}\left(W_{r}+W_{w}\right)=0$,

the growth/damping rate $\gamma$ is derived as (Misra and Tiwari, 1979)

$$
\frac{\gamma}{\Omega}=\frac{\frac{\Omega}{{ }_{\Pi} V_{T} \Pi}\left[\left(\frac{\Omega-\omega^{\prime}}{\Omega}\right)(J+1) \frac{V_{T \perp}^{2}}{V_{T}^{2}}-1\right] \exp \left[\begin{array}{c}
-\frac{\left(\omega^{\prime}-\Omega\right)^{2}}{k^{2} V_{T}^{2}} \\
\Pi
\end{array}\right]}{\left(\frac{c k}{\omega_{p i}}\right)^{2}\left(\frac{2 \Omega-\omega}{\Omega-\omega}\right)+\frac{1}{2}\left(\frac{2 \Omega-\omega^{\prime}}{\Omega-\omega^{\prime}}\right)^{2}}
$$

where $\omega^{\prime}=\omega-k_{\prod} V_{D}$ and the beam effect $\left(\mathrm{V}_{D}\right)$ appears through this term. Those particles with velocities near the phase velocity of the waves give up an energy $2 U$ to the waves. Half of this goes to potential energy and the other half goes into kinetic energy of the oscillation of the bulk of the particles.

Here it is noticed that the ion beam $V_{D}$ has affected the growth rate and change in the energy for the electromagnetic ion cyclotron (EMIC) waves propagating parallel to the magnetic field with a general loss-cone distribution function. The result is same as that derived by Kennel and Petschek (1966), if $J=0$ and effect of non-resonant particles is excluded. Thus, the transfer of energy to the particles, along with wave emission, is suitably described by this method of investigation.

\subsection{Marginal instability}

For the marginal instability condition $\gamma=0$, the maximum stable frequency is obtained

$$
K_{\Pi} V_{D}=\left[\omega-\Omega_{i}\left(1-\frac{1}{(J+1)} \frac{V_{T \amalg}^{2}}{V_{T \perp}^{2}}\right)\right] .
$$

The suitability of the particle aspect analysis to the magnetospheric dynamics lies in the evaluation of particle energies, along with the discussion on wave emission. The particle acceleration and wave emission are both significant to auroral electrodynamics. 


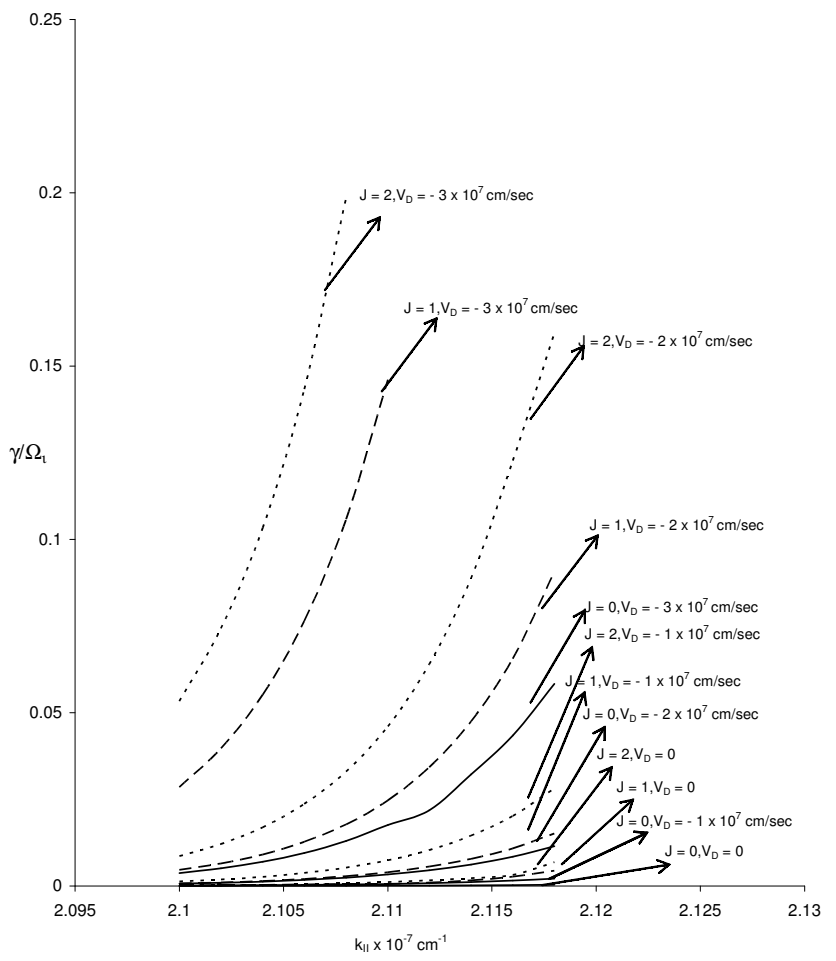

Fig. 1. Variation of growth rate $\left(\gamma / \Omega_{i}\right)$ versus wave vector $\mathrm{k}_{I I} \mathrm{~cm}^{-1}$ for different values of ion beam velocity $\mathrm{V}_{D}$, and distribution index $\mathrm{J}=0,1,2, \mathrm{~A}=15$.

In this analysis of the parallel propagating electromagnetic ion-cyclotron wave in the presence of a general losscone distribution function, we have derived the results for a bi-Maxwellian distribution and have replaced the anisotropy factor $A_{\max }=\frac{T_{\perp}}{T_{U}}-1$ of a bi-Maxwellian by the effective anisotropy factor $A_{\mathrm{eff}}=(J+1) \frac{V_{T \perp}^{2}}{V_{T}^{2}}-1$ in the presence of a general loss-cone distribution, as discussed in Gomberoff and Cuperman (1981); Summers and Thorne (1995). Thus, the effect of the loss-cone index $J$ is to increase the growth rate, as discussed in past (Ahirwar et al., 2006). In this paper the effects of the ion beam are emphasized in the presence of a general loss-cone distribution function, in the presence of EMIC waves.

\section{Results and discussion}

The role of the EMIC wave particle interaction in the auroral acceleration region is examined in the present analysis. In the auroral region EMIC wave instability is determined by (1) calculating the growth rates through the dispersion relation, using the auroral plasma parameters, (2) calculating the effects of the ion beam velocities on EMIC wave amplification with a general loss-cone distribution function and (3) studying the effects of thermal anisotropies on EMIC waves within the effect of a general loss-cone distribution function.

The characteristics of the EMIC waves were derived by using auroral acceleration parameters (Tiwari and Rostoker, 1984; Dwivedi et al., 2002; Mishra and Tiwari, 2006; Ahirwar et al., 2006) as

$$
\begin{aligned}
& B_{0}=4300 \mathrm{nTat} 1.4 R_{E} ; \quad \Omega_{i}=412 \mathrm{~s}^{-1} ; \\
& V_{T \| i}=6.41 \times 10^{8} \mathrm{~cm} / \mathrm{s} ; \quad \omega_{p i}^{2}=1.732 \times 10^{6} \mathrm{~s}^{-2} .
\end{aligned}
$$

The effects of the ion beam velocity $\left(\mathrm{V}_{D}\right)$, thermal anisotropy defined by $\mathrm{A}=\frac{V_{T \perp}^{2}}{V_{T}^{2}}$ with different distribution indices $J$ on the waves' growth rate and resonant energies are shown by Figs. 1 to 6.

Figure 1 shows the variation of the growth rate $\left(\gamma / \Omega_{i}\right)$ with the wave vector $\mathrm{k}_{\|}\left(\mathrm{cm}^{-1}\right)$ for different values of ion beam velocities $\mathrm{V}_{D}$ at $\mathrm{J}=0,1,2$, respectively. It is assumed that the ion beam is directed from the ionosphere towards the magnetotail and therefore is negative. It is observed that the effect of increasing the ion beam velocity is to enhance the growth rate that may be due to the shifting of the resonance condition. The ion beam energy is transferred to the wave, leading to the wave growth. The effect of higher distribution indices is to enhance the growth rate. Thus, the mirror-like structure of the magnetosphere with a steep loss-cone distribution index may be unstable for the EMIC waves emission. The growth rate increases with $\mathrm{k}_{\|}$in all cases.

The auroral acceleration region accelerates both ions upward along magnetic field lines, and electrons downward, and is, of course, better known because it creates the visible aurora. Ions, on the other hand, with a few but interesting exceptions, are mostly accelerated upwards. Ions that do precipitate in the energy exchange process are predominantly due to the direct injection into the loss cone in the region which we consider here as the auroral acceleration region. The average properties of upgoing ions, as seen by the DE-1 satellites, were described by Yau et al. (1985). Though auroral acceleration processes occur to some extent along the whole field line, measurements from S3-3, Viking and DE satellites have established that a large portion of the particle acceleration on auroral field lines occurs in an altitude range of about $1000 \mathrm{~km}$ to about $10000 \mathrm{~km}$. The simple explanation for this is that it is in this region that the relative drift velocities between ions and electrons are maximum, since the field-aligned currents increase in proportion to the magnetic field but the plasma density is still small. Thus acceleration is required either to maintain the current, or the large relative drift of particles can lead to EMIC instabilities and waves.

Figure 2 depicts the variation of transverse resonant energy $\left(\mathrm{W}_{r \perp}\right)$ erg $\mathrm{cm}$ versus wave vector $\mathrm{k}_{\|}\left(\mathrm{cm}^{-1}\right)$ for different values of ion beam velocities $\mathrm{V}_{D}$ at $\mathrm{J}=0,1,2$, respectively. The negative $\mathrm{W}_{r \perp}$ indicates that the particles' energy is transferred to the waves. Thus, wave emissions occur by extracting energy from the ions moving perpendicular to the 


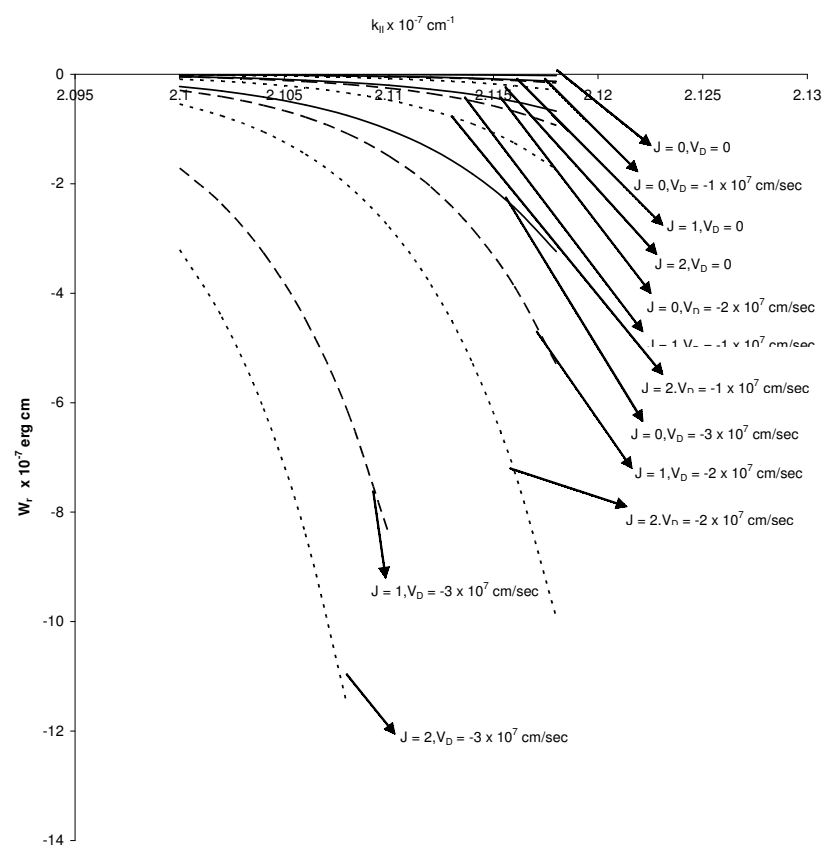

Fig. 2. Variation of perpendicular resonant energy $\mathrm{W}_{r \perp} \mathrm{erg} \mathrm{cm}$ versus wave vector $\mathrm{k}_{I I} \mathrm{~cm}^{-1}$ for different values of ion beam $\mathrm{V}_{D}$, and distribution index $J=0,1,2, A=15$.

magnetic field. It is observed that the effect of increasing $\mathrm{V}_{D}$ is to enhance the transfer of the particles' energy perpendicular to the magnetic field to the waves. Thus, the perpendicular deceleration of ions is noticed through the EMIC wave by ion beam energy. The effect of increasing values of the distribution indices is to increase the reduction in resonant energy. Thus, the steep loss-cone distribution of the magnetosphere decreases the transverse resonant energy in the presence of EMIC waves. It is also seen that the $\mathrm{W}_{r \perp}$ decreases with the increasing value of $\mathrm{k}_{\|}$. Thus, at higher $\mathrm{k}_{\|}$the growth rate and reduction in perpendicular energization are both enhanced, as the ion beam energy is being transferred to the waves. The upflowing ion beam may excite the wave, whereas it grows at the expense of the energy of the perpendicularly heated ions. The energy transfer to the waves may occur due to the cyclotron motion of the ions. The total energy of the ions rapidly decreases and they become subject to the $\nabla \mathrm{B}$ or mirror force, by means of which they are ejected from the perpendicular energization. The wave excitation is possible, as the transversely accelerated ions indicate. The transverse acceleration of ionospheric ions is a usual and normal process in the high latitude ionosphere, ongoing at all times over a wide area that is approximately coincident with the auroral oval. Recently, transversely accelerated ions and their association with EMIC waves have been reported by the analysis of FAST observations (Lund et al., 2000). The steep loss-cone decreases the heating rate of the transversely accelerated ions through the EMIC instability in the auroral acceleration region.

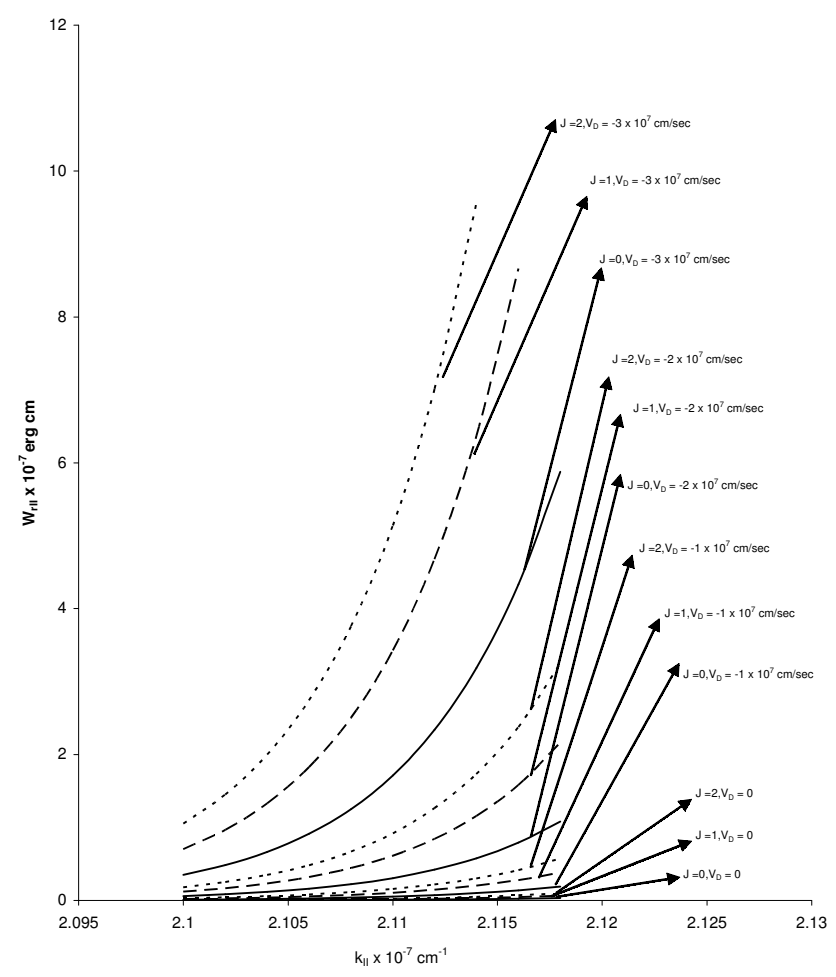

Fig. 3. Variation of parallel resonant energy $\mathrm{W}_{r I I} \mathrm{erg} \mathrm{cm}$ versus wave vector $\mathrm{k}_{I I} \mathrm{~cm}^{-1}$ for different values of ion beam $\mathrm{V}_{D}$, and distribution index $J=0,1,2, A=15$.

Figure 3 shows the variation of parallel resonant energy $\mathrm{W}_{r \| \mid}$ erg $\mathrm{cm}$ versus wave vector $\mathrm{k}_{\|}\left(\mathrm{cm}^{-1}\right)$ for different values of ion beam velocities $\mathrm{V}_{D}$ at $\mathrm{J}=0,1,2$, respectively. It is seen that the effect of $\mathrm{V}_{D}$ is to increase the parallel resonant energy. Thus, the ion beam may enhance the heating of resonant ions parallel to magnetic field, too. The effect of increasing values of the distribution indices is to increase the parallel resonant energy. Thus, the steep loss-cone distribution of the magnetosphere enhances the parallel resonant energy by the EMIC waves. The process occurs at the cost of ion beam energy. The enhancement of $\mathrm{Wr}_{\|}$, due to steep loss-cone distribution, has been reported previously by Ahirwar et al. (2006). The steep loss-cone structures are analogous to mirror-like devices with a higher mirror ratio which may accelerate the charged particles moving along the magnetic field (Varma and Tiwari, 1993; Dwivedi et al., 2002). Thus, the ion acceleration along the magnetic field is predicted by EMIC waves along the auroral field lines.

Upgoing, counterstreaming and downgoing field-aligned electron beams are often associated with upflowing ions with conical pitch angle distribution (i.e. ion conics) over the auroral region (Lund et al., 1999a, 2000, 2001; Miyake et al., 1998). Field-aligned electron beams are also found with upflowing ion beams in the auroral acceleration region (Hultqvist et al., 1991; Lundin and Eliasson, 1991; Yoshioka et al., 2000). The electromagnetic ion-cyclotron waves 


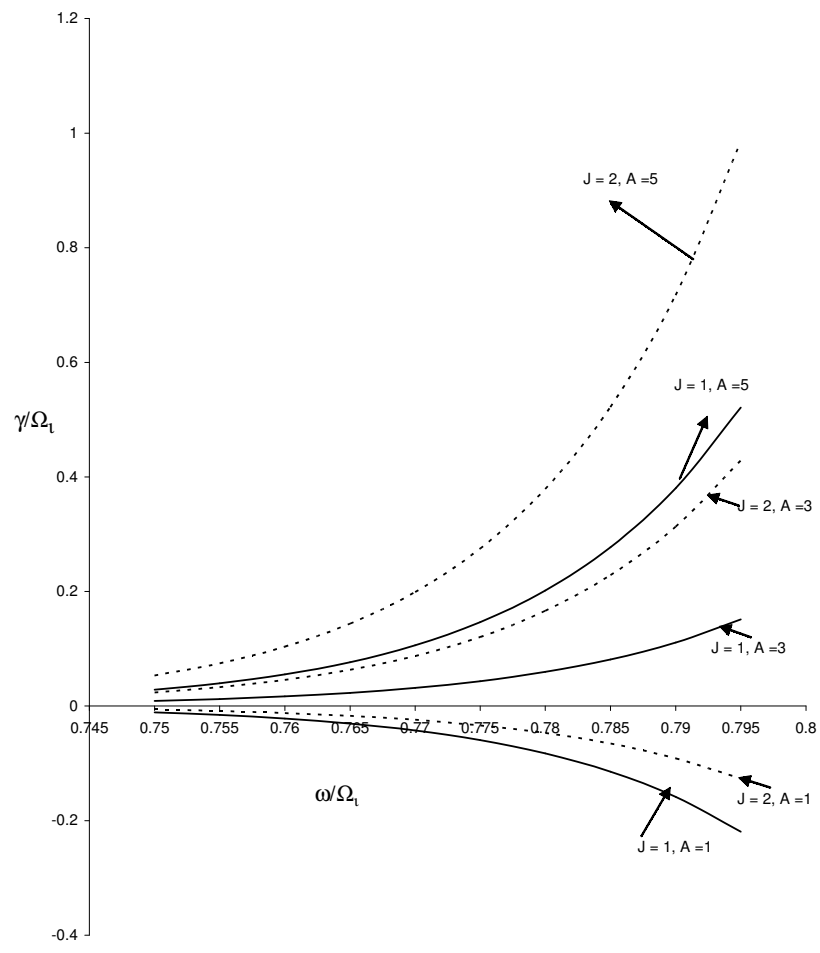

Fig. 4. Variation of growth rate $\left(\gamma / \Omega_{i}\right)$ versus wave frequency $\left(\omega / \Omega_{i}\right)$ for different values of thermal anisotropy (A) and distribution index $J=1,2, \mathrm{~V}_{D}=-3 \times 10^{7} \mathrm{~cm} / \mathrm{s}$.

generate a field-aligned electron beam (Temerin et al., 1986), but they accelerate electrons downward. The electromagnetic ion cyclotron waves are excited by electron beams of inverted-V structures (Erlandson and Zanetti, 1998). In the present investigation it is noted that EMIC waves are also generated by ion beams opposite to the direction of the propagation of the EMIC wave, however, these are damped by ion beams along the direction of propagation.

Figure 4 shows the variation of growth rate $\gamma / \Omega$ versus $\omega / \Omega_{i}$ for different values of thermal anisotropies A for a fixed value of $\mathrm{V}_{D}=-3 \times 10^{7} \mathrm{~cm} / \mathrm{s}$ at $\mathrm{J}=1$ and 2 , respectively. It is seen by this figure that the thermal anisotropies have enhanced the growth rate of EMIC waves with increasing values of distribution index $J$. The effect of thermal anisotropy during the substorm periods is to enhance the EMIC waves' emission. The collisionless wave-particle scattering by electromagnetic cyclotron fluctuations is a possible explanation for these anisotropies. The electromagnetic cyclotron frequency has also been observed in the solar wind (Tsurutani et al., 1994; Leamon et al., 1998; Goldstein and Roberts, et al., 1999) as a high-frequency excitation of the large-amplitude, low frequency electromagnetic fluctuations, which are ubiquitous in that medium (Coleman, 1968; Goldstein et al., 1994). In particular, Marsch and Tu (2001) and Tu and Marsch (2001) have demonstrated that velocity distribution is observed from the Helios spacecraft, which ex-

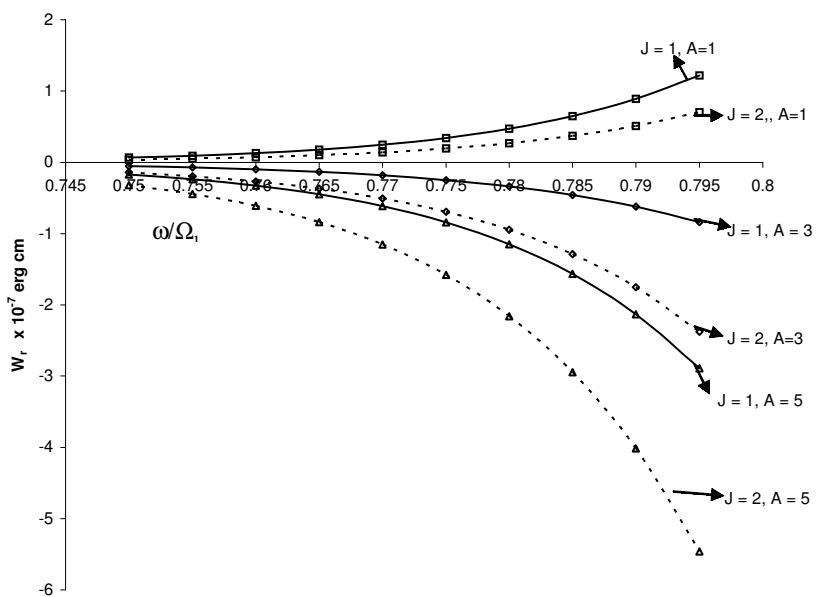

Fig. 5. Variation of perpendicular resonant energy $\mathrm{W}_{r \perp} \mathrm{erg} \mathrm{cm}$ versus wave frequency $\left(\omega / \Omega_{i}\right)$ for different values of anisotropy (A) and distribution index $J=1,2, \mathrm{~V}_{D}=-3 \times 10^{7} \mathrm{~cm} / \mathrm{s}$.

hibits anisotropies consistent with pitch angle scattering, by electromagnetic ion-cyclotron waves propagating away from the Sun. No growth rate is noticed in isotropic $(\mathrm{A}=1)$ plasma even at higher distribution indices $J$.

Figure 5 shows the variation of perpendicular resonant energy $\mathrm{Wr}_{\perp}$ erg cm versus $\omega / \Omega_{i}$ for different values of thermal anisotropies A for a fixed value of $\mathrm{V}_{D}=-3 \times 10^{7} \mathrm{~cm} / \mathrm{s}$ at $J=1$ and 2 , respectively. It is observed that by increasing the values of the thermal anisotropies A is to decrease the resonant transverse energy by the EMIC waves. The effect of increasing the values of the distribution indices is to decrease the $\mathrm{Wr}_{\perp}$, as well. Thus, the steep loss-cone distribution of the magnetosphere decreases the transverse energy as the wave frequency approaches the cyclotron frequency. It is also seen that the $\mathrm{Wr}_{\perp}$ decreases with the increasing values of $\omega / \Omega_{i}$, which implies that the energy is transferred from the particles to the EMIC waves. The direction reversal in $\mathrm{Wr}_{\perp}$, due to lower $\mathrm{A}$, is also noticed in the figure and that this is due to the damping of the EMIC waves (Fig. 4). Thus, the perpendicular heating of ions in the presence of a EMIC wave depends upon thermal anisotropy of the ions and loss-cone distribution indices.

Figure 6 shows the variation of parallel resonant energy $\mathrm{Wr}_{\|}$erg $\mathrm{cm}$ versus $\omega / \Omega_{i}$ for different values of thermal anisotropies A for fixed values of $\mathrm{V}_{D}=-3 \times 10^{7} \mathrm{~cm} / \mathrm{s}$ at $J=1$ and 2 , respectively. It is noticed that the effect of increasing the values of the distribution indices is to increase the parallel resonant energy, along with an increase in A. Thus, the steep loss-cone distribution of the magnetosphere may determine the parallel resonant energy of EMIC waves according to the frequency of the waves. The effect becomes maximum as the wave frequency approaches the cyclotron frequency at higher $J$ values. Therefore, the steep loss-cone distribution enhances the waves emission of the EMIC modes, as well as the heating of the ions parallel to the magnetic field, by 
extracting the energy of the perpendicularly heated ions. The mirroring force may become operative in association with EMIC waves, in order to control the heating and to emit the wave. If the $\mathrm{A}$ of an ion species becomes sufficiently large, it will excite an electromagnetic ion cyclotron anisotropy instability.

Observations made by the Freja satellites revealed detailed characteristics of the ion heating and corresponding wave phenomena at an altitude around $1700 \mathrm{~km}$. Norqvist et al. (1996) examined particle and wave data, and discussed the possible heating mechanism. They concluded that broadband waves around the ion cyclotron frequencies are the main energy source for the transversely accelerated ions and that the other mechanisms, such as lower hybride wave or a slowly varying electric field, are less important at an altitude around $1700 \mathrm{~km}$. Here we state that the perpendicular heating is reduced by EMIC waves in the presence of an ion beam in a mirror-like structure of the auroral ionosphere.

Electromagnetic wave ion beam plasma interactions take place in several space and astrophysics environments, as well as in laboratory plasmas. In the linear theory these waves have been studied both numerically and analytically (Gomberoff and Elgueta, 1991) in multi-component plasmas. The findings of the investigation may be of importance to the coronal heating and acceleration of solar wind by electromagnetic ion-cyclotron waves (e.g. Dusenbery and Hollweg, 1981; Shukla and Stenflo, 1985; Cranmer and Van Ballegooijen, 2003; Hollweg, 2006). It has been predicted that perpendicular heating of coronal ions is due to the dissipation of EMIC wave energy. However, the generation of EMIC waves has not been predicted yet on a firm basis. Since the mid-1990s, Solar and Helliospheric Observatory has predicted remarkable data and given impetus to studies on the ion-cyclotron resonance as the principal mechanism for heating the coronal holes, and ultimately driving the fast wind (Hollweg, 2006). In the solar corona, the ions are reported with higher temperatures than the electrons. The differential ion heating could be caused by large amplitude EMIC waves.

Amongst the various theories proposed for EMIC wave generation, our model may support the idea that rapid acceleration of fast solar wind in coronal holes is due mainly to the pressure tensor of the hot protons, which is primarily the magnetic mirror force, if the protons are highly anisotropic (Hollweg, 2006). Hollweg (2006) has stated that the non-Maxwellian particle distribution functions observed by spacecraft are trying to tell us something about the physics of heating and acceleration closer to the Sun, but their message has not been decoded. The model proposed by Hollweg (2006) of the inward, as well as outward propagating EMIC waves in the presence of ion beams oppositely directed to the wave propagation direction may be emphasized on the basis of the present findings. The ions may be perpendicularly heated by a low frequency MHD turbulence cascade (Shukla and Stenflo, 1985; Cranmer and Van Ballegooijen, 2003), generating the EMIC waves.

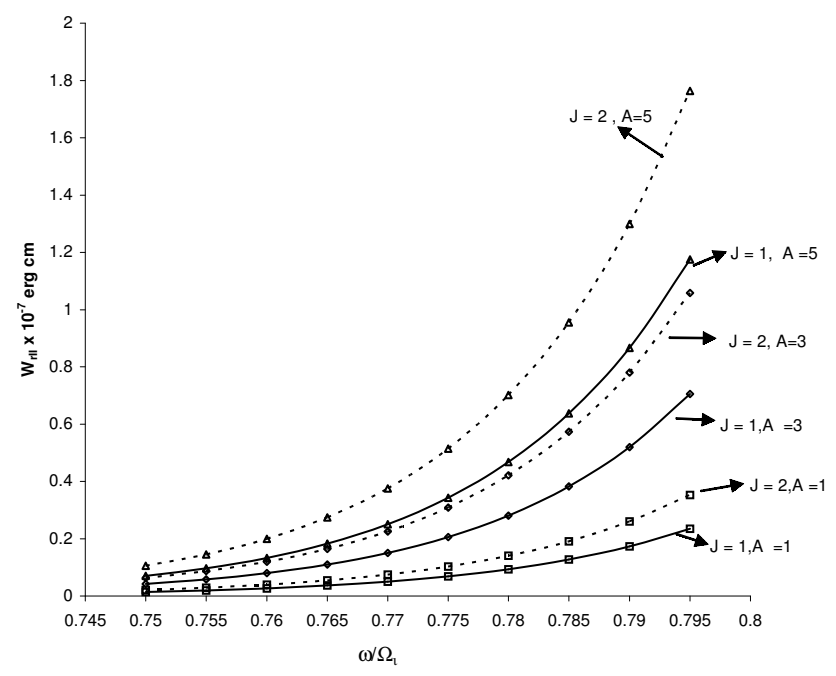

Fig. 6. Variation of parallel resonant energy $\mathrm{W}_{r I I} \mathrm{erg} \mathrm{cm}$ versus wave frequency $\left(\omega / \Omega_{i}\right)$ for different values of anisotropy (A) and distribution index $J=1,2, \mathrm{~V}_{D}=-3 \times 10^{7} \mathrm{~cm} / \mathrm{s}$.

Anderson et al. (1992a), studied the occurrence distribution of EMIC waves in the equatorial magnetosphere. They identified two regions, the early afternoon region $(\mathrm{L}>7, \mathrm{MLT}=11: 00$ to $15: 00)$ and the dawn region $(\mathrm{L}>7$, MLT=03:00 to 09:00), where there is a high occurrence rate of EMIC waves. They suggested that the predominance of $\mathrm{L}>7$ events implies that plasma sheet ion distributions develop sufficient temperature anisotropy to generate EMIC waves on a routine basis in their drift from the nightside to the dayside and that the plasma sheet ions open drift paths may present the greatest energy source for equatorially generated EMIC waves.

The theory may be useful to study the electrodynamics of the auroral ionospheric region. The EMIC turbulence may play an important role in the loss-cone current-potential relationship. It is also suggested that the loss-cone effect can enhance the anomalous resistivity for a given turbulence level. Since the steep loss-cone distribution in the presence of EMIC waves and the ion beam enhances the growth rate, the anomalous resistivity and transport resulting from this instability is likely to play a crucial role in the auroral acceleration region. The converging magnetic field lines in the higher latitude auroral ionosphere may be considered suitable for the use of a generalized loss-cone distribution function. An upflowing ion beam in a steep loss-cone may excite EMIC waves. The study may also be useful for the experimental devices with current carrying plasma (Varma and Tiwari, 1992). The particle aspect analysis developed may be applicable to laboratory plasma, as well as to estimate the heating rates, along with the study of emissions of EMIC waves.

Destabilizing effects due to the steep loss-cone on different instabilities are also reported by various workers (Tiwari and Varma, 1993; Varma and Tiwari, 1992; Baronia and 
Tiwari, 1999). The steep loss-cone structures are analogous to mirror-like devices with a higher mirror ratio, which may accelerate the charged particles perpendicular to the magnetic field and that may be a free energy source to excite EMIC waves. Thus, more energetic particles may be available to provide energy to the wave by wave-particles interactions.

\section{Conclusion}

In this paper we have conducted a comprehensive mathematical analysis and found how an electromagnetic ion-cyclotron wave may grow through the inverse Landau damping with an ion beam and thermal anisotropy. The effects of a general loss-cone distribution function are also incorporated in the auroral acceleration region to discuss EMIC waves emission. The concluding remarks of this study are as follows:

1. It is found that the effect of increasing ion beam velocity is to enhance the growth rate that may be due to a shifting of the resonance condition. The effect of higher distribution indices is to enhance the growth rate. The mirror-like structure of the magnetosphere with a steep loss-cone distribution may be unstable for the EMIC wave emission. The growth rate increases with $\mathrm{k}_{\|}$in all cases.

2. The steep loss-cone decreases the energy of the transversely accelerated ions through the EMIC instability in the auroral acceleration region in the presence of upflowing ion beam.

3. The effect of increasing the values of the distribution indices is to increase the parallel resonance energy. Thus, the steep loss-cone distribution of the magnetosphere enhances the parallel resonant energy by the EMIC waves in the presence of an upflowing ion beam.

4. The effect of thermal anisotropy during the substorm periods is to enhance the EMIC waves emission.

5. The energy of ions in the presence of EMIC waves depends upon the thermal anisotropy of the ions and losscone distribution indices in the presence of an upflowing ion beam.

6. The effect of increasing the values of the distribution indices is to increase the parallel resonant energy, along with an increase by A.

7. The findings may be applicable to explain EMIC wave emission and ion heating in the solar corona and the acceleration of the solar wind.

Acknowledgements. One of authors (P. Varma) is thankful to Department of Science \& Technology (DST), New Delhi for financial assistance.

Topical Editor I. A. Daglis thanks L. Gomberoff and another referee for their help in evaluating this paper.

\section{References}

Ahirwar, G., Varma, P., and Tiwari, M. S.: Electromagnetic ioncyclotron instability in the presence of parallel electric field with general distribution function-Particle aspect analysis, Ann. Geophys., 24, 1919-1930, 2006, http://www.ann-geophys.net/24/1919/2006/.

Anderson, B. J., Erlandson, R. E., and Zanetti, L. J.: A statistical study of Pc 1-2 magnetic pulsations in the equatorial magnetosphere 1. Equatorial occurrence distributions, J. Geophys. Res., 97, 3075-3088, 1992a.

Anderson, B. J. and Fuselier, S. A.: Response of thermal ions to electromagnetic ion Cyclotron waves, J. Geophys. Res., 99, 19413-19425, 1994.

Baronia, A. and Tiwari, M. S.: Particle aspect analysis of Alfvén wave, Ind. J. Phys., 73B(3), 499-507, 1999.

Cattell, C. A., Mozer, F. S., Roth, I., Anderson, R. R., Elphic, R. C., Lennartsson, W., and Ungstrup, E.: ISEE 1 observations of electrostatic ion cyclotron waves in association with ion beams on auroral field lines from $\sim 2.5$ to $4.5 \mathrm{Re}$, J. Geophys. Res., 96, $11421-11439,1991$.

Chaston, C. C., Ergun, R. E., Delory, G. T., et al.: Characteristics of electromagnetic proton cyclotron waves along auroral field lines observed by FAST in regions of upward current, Geophys. Res. Lett., 25, 2057-2064, 1998.

Chaston, C. C., Carlson, C. W., Ergun, R. E., and McFadden, J. P.: FAST observations of inertial Alfvén waves in the dayside aurora., Geophys. Res. Lett., 26, 647-650, 2002.

Coleman Jr., P. J.: Turbulence, viscosity and dissipation in the solar wind plasma, Astrophys. J., 153, 371-388, 1968.

Cranmer, S. R. and Van-Ballegooijen, A. A.: Alfvénic turbulence in the extended solar corona: Kinetic effects and proton heating, Astrophys. J., 594, 573-591, 2003.

Dawson, J.: On Landau Damping, Phys. Fluids, 4, 869-874, 1961.

Duan, S. P., Li, Z. Y., and Liu, Z. X.: Kinetic Alfvén wave driven by the density inhomogeneity in the presence of loss-cone distribution function-Particle aspect analysis, Planet. Space Sci., 53, 1167-1173, 2005.

Dusenbery, P. B. and Hollweg, J. V.: Ion-cyclotron heating and acceleration of solar wind minor ions, J. Geophys. Res., 86, 153 164, 1981.

Dwivedi, A. K., Varma, P., and Tiwari, M. S.: Kinetic Alfvén wave in the inhomogeneous magnetosphere and general distribution function, Planet. Space Sci., 49, 993-1003, 2001a.

Dwivedi, A. K., Varma, P., and Tiwari, M. S.: Kinetic Alfvén wave in the presence of parallel electric field in an inhomogeneous magnetosphere, Ind. J. Phys., 75(6), 555-563, 2001 b.

Dwivedi, A. K., Varma, P., and Tiwari, M. S.: Ion and electron beam effects on kinetic Alfvén wave in an inhomogeneous magnetosphere, Planet. Space Sci., 50, 93-99, 2002.

Erlandson, R. E. and Zanetti, L. J.: A statistical study of auroral electromagnetic ion cyclotron waves, J. Gesophys. Res., 103, 4627-4636, 1998.

Fuselier, S. A. and Anderson, B. J.: Low-energy $\mathrm{He}^{+}$and $\mathrm{H}^{+}$distributions and proton cyclotron waves in the afternoon equatorial magnetosphere, J. Geophys. Res., 101, 13 255-13 265, 1996.

Gendrin, R. and Roux, A.: Energization of helium ions by protoninduced hydromagnetic waves, J. Geophys. Res., 85, 4577-4586, 1980.

Goldstein, M. L., Roberts, D. A., and Fitch, C. A.: Properties of 
the fluctuating magnetic helicity in the inertial and dissipation ranges of solar wind turbulence, J. Geophys. Res., 99, 11519$11538,1994$.

Goldstein, M. L. and Roberts, D. A.: Magnetohydrodynamic turbulence in the solar wind, Phys. Plasma, 6, 4154-4160, 1999.

Gomberoff, L.: Stabilization of linear ion beam right hand polarized instabilities by nonlinear Alfvén/ion-cyclotron waves, J. Geophys. Res., 108, 1261, doi:1029/2003JA009837, 2003.

Gomberoff, L. and Cuperman, S.: On the kinetic instabilities of uniform magnetized plasmas with generalized loss-cone distribution function, J. Plasma Phys., 25, 99-102, 1981.

Gomberoff, L. and Neira, R.: Convective growth rate of ion cyclotron waves in a $\mathrm{H}^{+} / \mathrm{He}^{+}$and $\mathrm{H}^{+} / \mathrm{He}^{+} / \mathrm{O}^{+}$plasma, J. Geophys. Res., 88, 2170-2174, 1983.

Gomberoff, L. and Elgueta, R.: Resonant acceleration of alpha particles by ion-cyclotron waves in the solar wind, J. Geophys. Res., 96, 9801-9804, 1991.

Gomberoff, L., Gratton, F. T., and Gnavi, G.: Acceleration and heating of heavy ions by circularly polarized Alfvén waves, J. Geophys. Res., 101, 15 661-15 666, 1996.

Hollweg, J. V.: Drives of the solar wind: then and now, Phil. Trans. R. Soc. A., 364, 505-527, 2006.

Horne, R. B. and Thorne, R. M.: Wave heating of $\mathrm{H}_{e}^{+}$by electromagnetic ion-cyclotron waves in the magnetosphere: heating near the $\mathrm{H}^{+}-\mathrm{H}_{e}^{+}$bi-ion resonance frequency, J. Geophys. Res., 102, 11 457-11471, 1997.

Horne, R. B. and Thorne, R. M.: Electron pitch angle diffusion by electrostatic electron cyclotron waves: the origin of pancake distributions, J. Geophys. Res., 105, 5391-5402, 2000.

Hultqvist, B., Vo, H., Lundin, R., Aparicio, B., Lindqvist, P.-A., Gustafsson, G., and Holback, B.: On the upward acceleration of electrons and ions by low-frequency electric field fluctuations observed by Viking, J. Geophys. Res., 96, 11 609-11 615, 1991.

Kennel, C. F. and Petschek, H. E.: Limit on stably trapped particle fluxes, J. Geophys. Res., 71, 1-28, 1966.

Leamon, R. J., Smith, C. W., Ness, N. F., Mathaeus, W. H., and Wong, H. K.: Observational constraints on the dynamics of the interplanetary magnetic field dissipation range, J. Geophys. Res., 103, 4775-4787, 1998.

Lundin, R. and Eliasson, L.: Auroral energization processes, Ann. Geophys, 9, 202-223, 1991.

Lund, E. J., Mobius, E., Klumpar, D. M., Kistler, L. M., Popecki, M. A., Klecker, B., Ergun, R. E., McFadden, J. P., Carlson, C. W., and Strangeway, R. J.: Occurrence distribution of preferentially heating events in the Aurora, Adv. Space Res., 23, 1721-1724, $1999 \mathrm{a}$.

Lund, E. J., Mobius, E., Klumper, D. M., Kistler, L. M., Popecki, M. A., Klecker, B., Ergun, R. E., McFadden, J. P., Carlson, C. W., and Strangeway, R. J.: Direct comparison of transverse ion acceleration mechanisms in the aurora region of solar minimum, J. Geophys. Res., 104, 22 801-22 805, 1999 b.

Lund, E. J., Mobius, E., Ergun, R. E., and Carlson, C. W.: Massdependent effects in ion conic production: The role of parallel electric fields, Geophys. Res. Lett., 26, 3593-3596, 1999c.

Lund, E. J., Mobius, E., Carlson, C. W., Ergun, R. E., Kistler, L. M., Klecker, B., Klumpar, D. M., McFadden, J. P., Popecki, M. A., Strangeway, R. J., and Tung, Y. K.: Transverse ion acceleration mechanism in the aurora at solar minimum: occurrence distributions, J. Atmos. Solar Terres. Phys., 62, 467-475, 2000.
Lund, E. J., Mobius, E., Lynch, K. A., Klumpar, D. M., Peterson, W. K., Ergun, R. E., and Carlson, C. W.: On the Mass Dependence of Transverse Ion Acceleration by Broad-Band Extremely Low Frequency Waves, Phys. Chem. Earth, 26, 161-163, 2001.

Marsch, E. and Tu, C.-Y.: Heating and acceleration of coronal ions interacting with plasma waves through cyclotron and landau resonance, J. Geophys. Res., 106, 227-238, 2001.

Mauk, B. H., Mcllwain, C. E., and Mcpherron, R. L.: Helium cyclotron resonance within the Earth's magnetosphere, Geophys. Res. Lett., 8, 103-106, 1981.

McFadden, J. P., Ergun, R. E., Elphic, R., et al.: Electron modulation and ion cyclotron waves observed by FAST, Geophys. Res. Lett., 25, 2045-2048, 1998a.

Misra, K. D. and Tiwari, M. S.: Particle aspect analysis of electromagnetic ion cyclotron Instability, Can. J. Phys., 57, 1124-1130, 1979.

Miyake, W., Mukai, T., and Kaya, N.: Astatistical study of fieldaligned electron beams associated with ion conics events, Ann. Geophys., 16, 940-947, 1998, http://www.ann-geophys.net/16/940/1998/.

Mishra, R. and Tiwari, M. S.: Effect of parallel electric field on electrostatic ion cyclotron instability in anisotropic plasma in the presence of ion beam and general distribution function, Planet. Space Sci., 52, 188-199, 2006.

Mozer, F. S., Carlson, C. A., Hudson, M. H., et al.: Observations paired electrostatic shocks in the polar magnetosphere, Phys. Rev. Lett., 38, 292-295, 1977.

Mozer, F. S. and Hull, A.: The origin and geometry \& upward parallel electric field in the auroral acceleration region, J. Geophys. Res., 106, 5763-5778, 2001.

Norqvist, P., Andre, M., Eliasson, L., et al.: Ion cyclotron heating in the dayside magnetosphere, J. Geophys. Res., 101, 13179 13 193, 1996.

Schriver, D., Ashour-Abdalla, M., Strangeway, R. J., Richard, R. L., Klezting, C., Dotan, Y., and Wygant, J.: FAST/Polar conjuction study of field-aligned auroral acceleration and corresponding magnetotail drivers, J. Geophys. Res., 108(A9), 8020, doi:10.1029/2002JA009426, 2003.

Sharp, R. D., Johnson, R. G., and Shelley, E. G.: Observation of an ionospheric acceleration mechanism producing energetic (kev) ions primarily normal to the geomagnetic field direction, J. Geophys. Res., 82, 3324-3328, 1977.

Shelley, E. G., Sharp, R. D., and Johnson, R. G.: Satellite observations of an ionospheric acceleration mechanism, Geophys. Res. Lett., 3, 654-656, 1976.

Shukla, P. K. and Stenflo, L.: Non-linear propagation of electromagnetic ion-cyclotron Alfvén waves, Phys. Fluids, 28, 1576$1578,1985$.

Summers, D. and Thorne, R. M.: Plasma microinstabilities driven by loss-cone distributions, J. Plasma Phys., 53, 293-315, 1995.

Terashima, Y.: Particle aspect analysis of drift instabilities, Prog. Theor. Phys., 37, 775-785, 1967.

Temerin, M. and Roth, I.: Ion heating by waves with frequencies below the ion gyrofrequency, Geophys. Res. Lett., 13, 1109-1115, 1986.

Temerin, M. and Lysak, R. L.: Electromagnetic ion cyclotron (ELF) waves generated by auroral electron precipitation, J. Geophys. Res., 89, 2849-2859, 1984.

Thorne, R. M. and Horne, R. B.: Modulation of electromagnetic 
ion cyclotron instability due to interaction with ring current $\mathrm{O}^{+}$ during magnetic storms, J. Geophys. Res., 102, 14 155-14 163, 1997.

Tiwari, M. S. and Rostoker, G.: Field aligned currents and auroral acceleration by non-linear MHD waves, Planet. Space Sci., 32, 1497-1503, 1984.

Tiwari, M. S. and Varma, P.: Drify instability in the presence of parallel electric field and an inhomogeneous magnetic field - Particle aspect analysis, J. Plasma Phys., 46, 49-62, 1991.

Tiwari, M. S. and Varma, P.: Drift wave instability with loss-cone distribution function - particle aspect analysis, Planet. Space Sci., 41, 199-207, 1993.

Tsurutani, B. T., Arballo, J. K., Mok, J., Smith, E. J., Mason, G. M., and Tan, L. C.: Electromagnetic waves with frequencies near the local proton gyrofrequency: ISEE-3 AU observations, Geophys. Res. Lett., 21, 633-636, 1994.
Tu, C.-Y. and Marsch, E.: On cyclotron wave heating and acceleration of solar wind ions the outer corona, J. Geophys. Res., 106, 8233-8252, 2001.

Varma, P. and Tiwari, M. S.: Ion and electron beam effects on drift wave instability with different distribution function - Particle aspect analysis, Phys. Scr. 45, 275-279, 1992.

Varma, P. and Tiwari, M. S.: Drift wave in the presence of an AC electric field with different distribution function-particle aspect analysis, Indian Journal of Pure Appl. Phys., 31, 616-623, 1993.

Yau, A. W., Shelley, E. G., Peterson, W. K., and Leuchyshyn, L.: Energetic auroral and polar on outflow at DE-1 altitudes: magnitude composition magnetic activity dependence and log-term variatios, J. Geophys. Res., 90, 8417-8432, 1985.

Yoshioka, R., Miyake, W., Mukai, T., and Ito, M.: Field-aligned electron beams observed simultaneously with upflowing ion beams in the auroral acceleration region, J. Geophys. Res., 105, 7679-7694, 2000. 